Check for updates

Cite this: Mater. Adv., 2020 1,1745

Received 22nd June 2020, Accepted 30th July 2020

DOI: 10.1039/d0ma00442a

rsc.li/materials-advances

\section{Fabrication of polydiacetylene particles using a solvent injection method $\dagger$}

\author{
Junwei Tang, ${ }^{a}$ Max Weston, ${ }^{a}$ Rhiannon P. Kuchel, ${ }^{b}$ Fabio Lisi, (D) ${ }^{a}$ Kang Liang (D) ac \\ and Rona Chandrawati (D) *a
}

\begin{abstract}
Polydiacetylenes (PDAs) are a class of conjugated polymers with unique optical properties that have practical applications in a diverse range of fields. PDA vesicular particles are typically constructed using a thin film hydration method which suffers from a lack of scalability and requires techniques such as sonication and extrusion to control particle size. These procedures are energy-intensive and limit the application of the thin film hydration method to small-scale vesicle production. We present the first application of the solvent injection method to synthesize PDA vesicles as an alternative method of high throughput production. PDA vesicles are constructed from a range of DA monomers that vary in their total carbon chain length and were analyzed using transmission electron microscopy, dynamic light scattering, and zeta potential measurements. Using the solvent injection method, we found longer alkyl chain length monomers produce smaller particles that are more easily photopolymerized. The function of the PDA is verified for the detection of ammonia gas. 8,10-Henicosadiynoic acid is nominated as the optimum PDA system as it has a moderate chain length which results in both optimum morphology and ammonia detection sensitivity. The results from this study suggest that the solvent injection method is a viable alternative to the thin film hydration method for large scale PDA particle production.
\end{abstract}

\section{Introduction}

Polydiacetylenes (PDAs) are a class of conjugated polymers exhibiting unique optical properties that render them ideal for the construction of colorimetric sensors to detect chemical and biological analytes. ${ }^{1-5}$ They are synthesized from diacetylene (DA) monomers that undergo photopolymerization (by $254 \mathrm{~nm}$ UV light) via a 1,4-addition reaction to generate a polymeric backbone with alternating $\mathrm{C}=\mathrm{C}$ and $\mathrm{C} \equiv \mathrm{C}$ bonds (ene-yne). Subjecting PDA to stimuli - such as light, heat, $\mathrm{pH}$ or a chemical/biological recognition event - can induce a perturbation in the polymer structure. This alters the effective conjugation length, producing a characteristic blue to red color transition that can be easily detected by the naked eye (a shift in absorbance wavelength from $640 \mathrm{~nm}$ (blue phase) to $540 \mathrm{~nm}$ (red phase)). Carboxy-terminated diacetylene monomers are amphiphilic and readily self-assemble into vesicles in solution. This orients the monomers favorably for photopolymerization and provides an

\footnotetext{
${ }^{a}$ School of Chemical Engineering and Australian Centre for Nanomedicine (ACN), The University of New South Wales (UNSW Sydney), Sydney, NSW 2052, Australia. E-mail: rona.chandrawati@unsw.edu.au

${ }^{b}$ Electron Microscope Unit, Mark Wainwright Analytical Centre, The University of New South Wales (UNSW Sydney), Sydney, NSW 2052, Australia

'Graduate School of Biomedical Engineering, The University of New South Wales (UNSW Sydney), Sydney, NSW 2052, Australia

$\dagger$ Electronic supplementary information (ESI) available. See DOI: 10.1039/d0ma00442a
}

excellent format for functionalization with recognition elements by insertion in or conjugation to the vesicle bilayer. ${ }^{1-9}$

The conventional method for synthesis of PDA vesicles is the thin film hydration method (Bangham method) which was developed in 1965 for the production of liposomes from phospholipids. ${ }^{10}$ Using this method, DA monomers are first dissolved in a suitable polar solvent, typically chloroform or ethanol. The solvent is then evaporated to leave a thin film of DA monomer. The film is hydrated at a temperature greater than the phase transition temperature of the monomer and a heterogeneous mixture of multilamellar vesicles (MLVs) is spontaneously formed. This method is fast and easy which has seen its widespread adoption in lab-scale synthesis of PDA vesicles. Unfortunately, the film hydration method suffers from several drawbacks that limit its application in industrial-scale synthesis. Heterogeneous mixtures of MLVs are undesirable so the vesicles are often subjected to extrusion, sonication, or high-pressure homogenization to produce monodispersed populations of small unilamellar vesicles (SUVs). These procedures can destroy incorporated recognition elements or vesicle cargo. They must also be conducted above the phase transition temperature of the monomers. As the phase transition temperature of DA monomers is quite high $\left(50-80{ }^{\circ} \mathrm{C}\right)$, these techniques become difficult and highly energy-intensive.

Herein, we propose the solvent injection method as a suitable alternative for the synthesis of PDA vesicles over the 


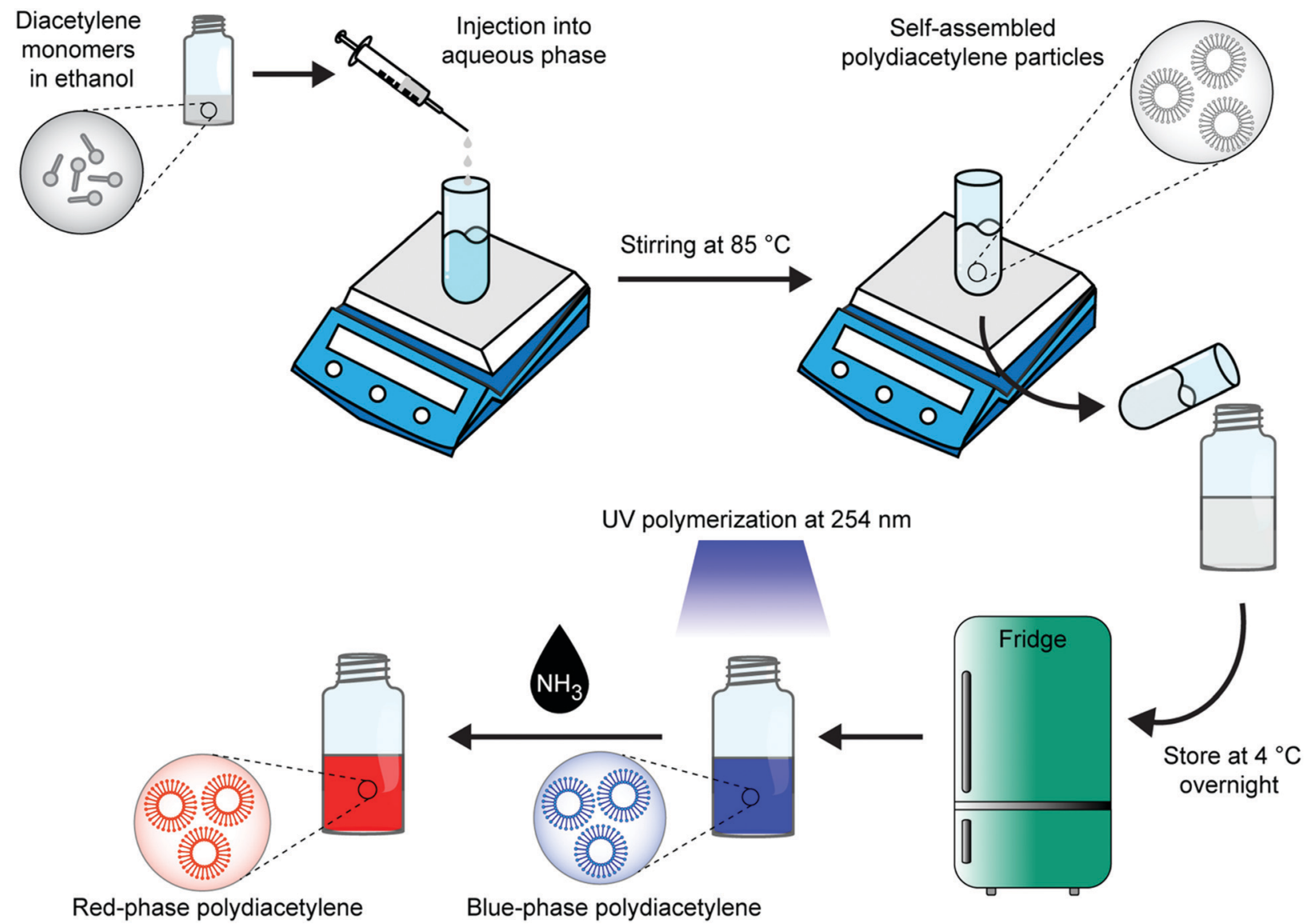

Fig. 1 Schematic illustration of polydiacetylene (PDA) vesicle synthesis using a solvent injection method. Diacetylene monomers are dissolved in a polar solvent (e.g., ethanol) and then slowly added to an aqueous medium at above the phase transition temperature of the monomer under vigorous stirring. The ethanol is evaporated and the amphiphilic diacetylene monomers self-assemble into vesicles. The diacetylene vesicles are left to anneal at $4{ }^{\circ} \mathrm{C}$ and then undergo photopolymerization by $254 \mathrm{~nm}$ UV light yielding blue-phase PDA. Subjecting PDA to stimulus, for example ammonia, induces a characteristic blue to red color transition that can be easily detected by the naked eye.

thin film hydration method (Fig. 1). The solvent injection method was first developed in 1973 to prepare liposomes, ${ }^{11}$ however its application to PDA vesicle synthesis has not yet been investigated. In this method, we first dissolve the DA monomers in a polar solvent (in this case ethanol). The DA solvent solution is then slowly added to an aqueous medium at above the phase transition temperature of the monomer under vigorous stirring. The ethanol is evaporated, and the amphiphilic diacetylenes selfassemble into vesicles. In the construction of liposomes, it has been demonstrated that control over solvent injection flow rate, lipid concentration, lipid type, and solvent ratio dictate the size and lamellarity of the vesicles. ${ }^{12-16}$ Optimization of these parameters can yield SUV liposomes without the need for secondary procedures as required in the film hydration method. As a result, this method shows great promise for large scale production of PDA vesicles.

Recently, we discussed how the selection of the DA monomer can be used to tune the chromatic response, sensitivity, and specificity of PDA sensors. ${ }^{1}$ Herein we present the first investigation of the solvent injection method for the construction of PDA vesicular particles and test its applicability to a range of DA monomers (Fig. 2). The monomers vary in their total carbon chain length, alkyl spacer length, and alkyl tail length. The success of this synthetic method is demonstrated by studying the formation and morphology of the particles, their ability to be photopolymerized, and their chromatic properties.

\section{Materials and methods}

\section{Materials}

Diacetylene monomers 10,12-pentacosadiynoic acid (PCDA) (>97\%), 10,12-tricosadiynoic acid (TCDA) (>98\%), and 5,7hexadecadiynoic acid (HDDA) (97\%) were purchased from SigmaAldrich (Australia). Diacetylene monomers 10,12-octadecadiynoic acid (ODDA) (95\%), 8,10-henicosadiynoic acid (HCDA) (97\%), and 6,8-nonadecadiynoic acid (NDDA) (96\%) were purchased from Fujifilm Wako Chemicals (USA). Ethanol absolute and ammonia $30 \%$ solution were purchased from Chem Supply (Australia). All chemicals were used as received without further purification. Ultrapure water (18.2 $\mathrm{M} \Omega \mathrm{cm}^{-1}$ resistance) was provided by arium ${ }^{\mathbb{R}}$ pro Ultrapure Water Systems (Sartorius).

\section{Synthesis of PDA vesicles}

DA monomers were dissolved in ethanol $\left(8 \mathrm{mg} \mathrm{ml}^{-1}, 500 \mu \mathrm{l}\right)$ and injected into a vial containing $10 \mathrm{ml}$ of water at $20 \mu \mathrm{min}^{-1}$ 
a) 10,12-Pentacosadiynoic acid (PCDA, 25 carbon atoms)

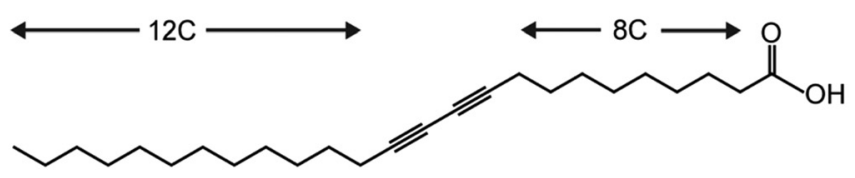

b) 10,12-Tricosadiynoic acid (TCDA, 23 carbon atoms)

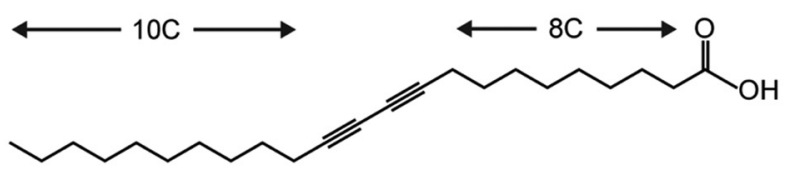

c) 10,12-Octadecadiynoic acid (ODDA, 18 carbon atoms)

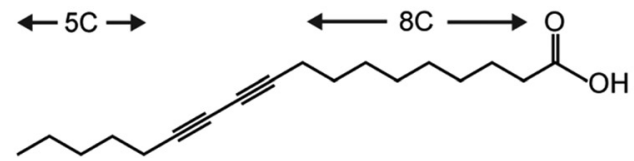

d) 8,10-Henicosadiynoic acid (HCDA, 21 carbon atoms)

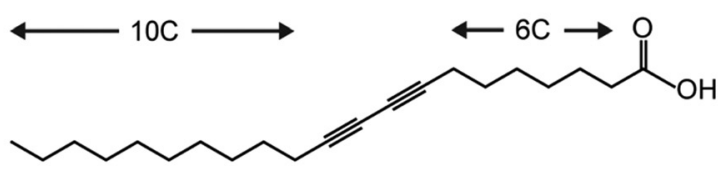

e) 6,8-Nonadecadiynoic acid (NDDA, 19 carbon atoms)

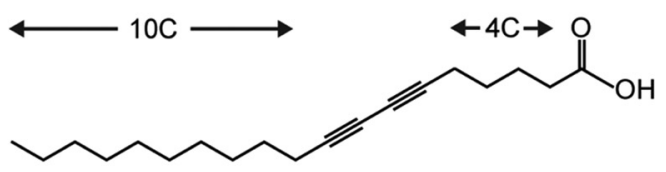

f) 4,6-Heptadecadiynoic acid (HDDA, 17 carbon atoms)

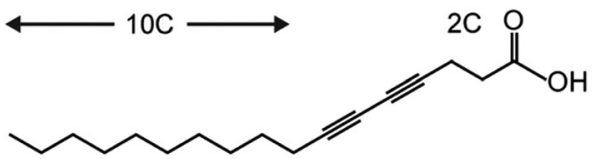

Fig. 2 Chemical structures of diacetylene monomers used to synthesize polydiacetylene in this study.

flow rate. During the solvent injection process, the solution was maintained at $85{ }^{\circ} \mathrm{C}$ under constant stirring at $1000 \mathrm{rpm}$. After the DA monomer injection step was complete, the DA/ethanol/ water mixture was incubated for further $1 \mathrm{~h}$ at $85{ }^{\circ} \mathrm{C}$ and $1000 \mathrm{rpm}$ to allow for ethanol evaporation (see ESI $\dagger$ ). The self-assembled DA vesicles in water (at $0.4 \mathrm{mg} \mathrm{ml}^{-1}$ monomer concentration) were allowed to cool to room temperature and then stored at $4{ }^{\circ} \mathrm{C}$ overnight. Photo-induced polymerization was carried out under $254 \mathrm{~nm}$ UV irradiation (UV lamp LF-206.LS, 6W, UVITEC, UK) for 5 minutes to obtain blue-phase PDA.

\section{PDA characterization}

Dynamic light scattering (DLS) and zeta potential. The size and surface charge of PDA particles were determined with a Zetasizer Nano ZS (Malvern, UK; $4 \mathrm{~mW}$ He-Ne laser, $\lambda_{0}=$ $633 \mathrm{~nm}, \theta=173^{\circ}$ ). To measure the size, $1 \mathrm{ml}$ of the PDA samples was transferred to a disposable cuvette (DTS0012) without dilution and equilibrated at room temperature for $5 \mathrm{~min}$. Each sample was measured three times, each measurement consisting of 6 individual runs. The values for the viscosity $(0.8872 \mathrm{cP})$ and refractive index (1.330) of the solvent were the ones provided by the Zetasizer software (V. 7.13). The data were analyzed using the general purpose non-negative least squares (NNLS) fitting algorithm, with a size range analysis of $0.4-10000 \mathrm{~nm}$. The vesicles' size and standard deviation were calculated from the intensity distributions. Zeta potential measurements were collected using a disposable folded capillary cell (DTS1070), containing $0.6 \mathrm{ml}$ of the sample without dilution.

Transmission electron microscopy (TEM). TEM micrographs were obtained using a JEOL1400 TEM (JEOL, Akishima, Japan) operating at an accelerating voltage of $100 \mathrm{keV}$. Samples were drop cast onto formvar-supported copper grids (ProSciTech, Australia) without staining. The size and morphology of the particles were recorded using a Phurona CCD Camera (Emsis) and Radius software (Emsis).
UV-vis spectrophotometer. The PDA solutions were transferred to 96-well plates and UV-vis absorption spectra were recorded with a SpectraMax M5 microplate reader (Molecular Devices, USA).

Colorimetric response analysis. The colorimetric response $(\mathrm{CR} \%)$ is the standard method to quantify blue-to-red color transition of $\mathrm{PDA}^{2}$ and is calculated as:

$$
\mathrm{CR} \%=\frac{\mathrm{PB}_{0}-\mathrm{PB}_{\mathrm{f}}}{\mathrm{PB}_{0}} \times 100 \%
$$

where $\mathrm{PB}_{0}$ and $\mathrm{PB}_{\mathrm{f}}$ are the initial and final percentages of blue (PB) of the PDA system, respectively. PB is defined as:

$$
\mathrm{PB}=\frac{A_{\text {blue }}}{A_{\text {blue }}+A_{\text {red }}} \times 100 \%
$$

where $A_{\text {blue }}$ is the absorbance value at the selected wavelength for the blue-phase PDA $(640 \mathrm{~nm})$ and $A_{\text {red }}$ is the absorbance value at the selected wavelength for the red-phase PDA $(540 \mathrm{~nm})$.

\section{Ammonia detection}

To verify the functionality of the PDA formed using the solvent injection method, we chose ammonia gas as an example of a target analyte. Ammonia $30 \% \mathrm{w} / \mathrm{w}$ solution was first diluted to ammonia $1 \% \mathrm{w} / \mathrm{w}$ solution with ultrapure water. Varying volume of ammonia $1 \%$ solution was added into glass containers $(600 \mathrm{ml})$ : $5.06 \mu \mathrm{l}(100 \mathrm{ppm}), 15.18 \mu \mathrm{l}(300 \mathrm{ppm}), 25.30 \mu \mathrm{l}(500 \mathrm{ppm}), 50.59 \mu \mathrm{l}$ (1000 ppm), $75.88 \mu \mathrm{l}(1500 \mathrm{ppm})$, and $101.18 \mu \mathrm{l}$ (2000 ppm). PDA solutions $(250 \mu \mathrm{l})$ in glass vials were exposed to ammonia gas in airtight glass containers and the color changes of the PDA were monitored over time. The gas phase concentration of ammonia was determined by the ideal gas law equation:

$$
P V=n R T
$$


where $P$ is the pressure ( $1 \mathrm{~atm}), V$ is the volume of ammonia gas (L), $n$ is the amount of ammonia gas (mol), $R$ is the ideal gas constant $\left(0.08206 \mathrm{~L} \mathrm{~atm} \mathrm{~mol}{ }^{-1} \mathrm{~K}^{-1}\right), T$ is the room temperature (298.15 K).

\section{Results and discussion}

The solvent injection method was employed to synthesize PDA particles from a range of DA monomers. To determine the method's efficacy, a study on the particle size and morphology by DLS and TEM was conducted to confirm colloid formation.

Table 1 Size (from the intensity plot), polydispersity index, and surface charge of PDA particles formed using a solvent injection method

\begin{tabular}{llll}
\hline Monomers & Diameter $(\mathrm{nm})$ & PDI & Zeta potential $(\mathrm{mV})$ \\
\hline PCDA (25C) & $127.2 \pm 58.3$ & $0.129 \pm 0.049$ & $-32.2 \pm 8.6$ \\
TCDA (23C) & $184.1 \pm 59.9$ & $0.068 \pm 0.023$ & $-29.0 \pm 6.8$ \\
HCDA (21C) & $281.9 \pm 91.3$ & $0.072 \pm 0.027$ & $-26.7 \pm 6.7$ \\
NDDA (19C) & $456.1 \pm 130.7$ & $0.141 \pm 0.044$ & $-32.0 \pm 7.1$ \\
ODDA (18C) & $800.3 \pm 237.1$ & $0.767 \pm 0.174$ & $-26.9 \pm 7.5$ \\
HDDA (17C) & Particle aggregation &
\end{tabular}

a)

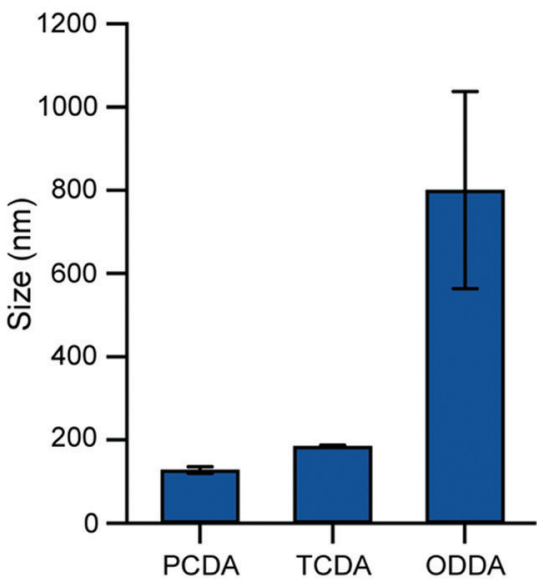

b)

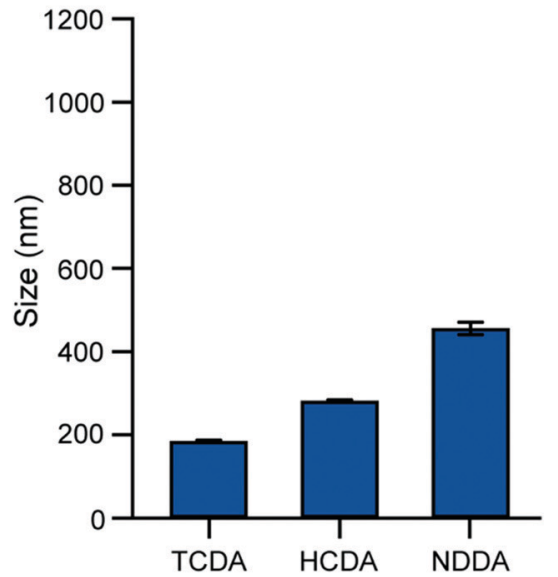

Fig. 3 (a) Size of PDA particles synthesized from monomers with the same alkyl spacer length and different alkyl tail length. (b) Size of PDA particles synthesized from monomers with different alkyl spacer length and the same alkyl tail length. $n=3$, values represent mean \pm standard deviation
Different amphiphilic DA monomer building blocks selfassembled to form vesicles of a characteristic size with their diameters ranging from $127.2 \mathrm{~nm}$ to $800.3 \mathrm{~nm}$ (Table 1). Longer chain monomers (PCDA 25C; TCDA 23C; HCDA 21C) formed smaller particles with a narrow size distribution (polydispersity index $(\mathrm{PDI}) \sim 0.1$ ) compared with shorter chain monomers (NDDA 19C; ODDA 18C). The longer chain monomers have larger intermolecular forces which have been shown to facilitate vesicle formation. ${ }^{17}$ Monomers with the same length alkyl spacers but longer alkyl tails formed smaller particles (Fig. 3a). Monomers with shorter alkyl spacers between the carboxylic acid head group and the diacetylene group formed larger particles (Fig. 3b). The positioning of the diacetylene group may impact monomer flexibility and its ability to form compact vesicles.

TEM micrographs were obtained to further investigate the morphology of the PDA structures (Fig. 4). Particles formed from long chain monomers (PCDA and TCDA) exhibited a relatively spherical shape, indicating homogenous ordering throughout the PDA structure. As for shorter chain monomers (HCDA and NDDA), rod-shaped and rectangular particles tended to form of a lesser order. ODDA formed both small particles under $100 \mathrm{~nm}$ as well as micron-sized structures (see ESI $\dagger$ ). These results indicate that a constant monomer concentration $\left(0.4 \mathrm{mg} \mathrm{ml}^{-1}\right)$ there is a relationship between monomer properties and particle morphology. The TEM micrographs indicate a morphology similar to particles synthesized by the film hydration method, ${ }^{18}$ confirming the efficacy of the solvent injection method for PDA synthesis.

DA monomers are amphiphilic and are known to form a thermodynamically favorable bilayer/vesicular structure in an
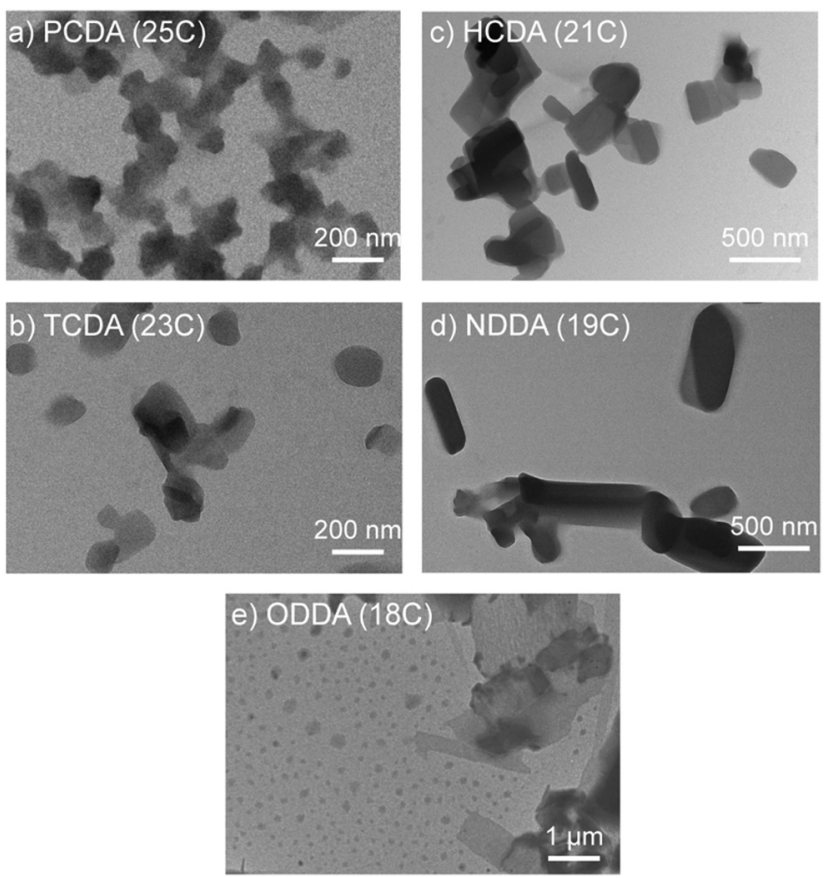

Fig. 4 TEM micrographs of PDA particles synthesized from 5 diacetylene monomers using the solvent injection method. (a) PCDA ( 25 carbon atoms), (b) TCDA (23 carbon atoms), (c) HCDA (21 carbon atoms), (d) NDDA (19 carbon atoms), and (e) ODDA (18 carbon atoms). 
aqueous environment. Despite some disagreement between the exact boundaries of vesicle classification, typically particles with a size range between $20 \mathrm{~nm}$ and $100 \mathrm{~nm}$ are considered small unilamellar vesicles (SUVs), whilst particles ranging a)
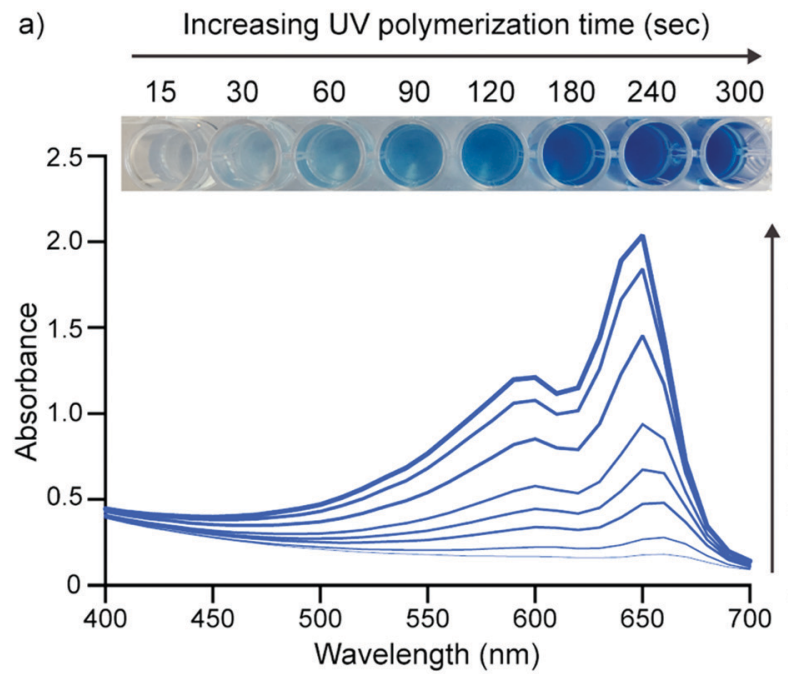

c)
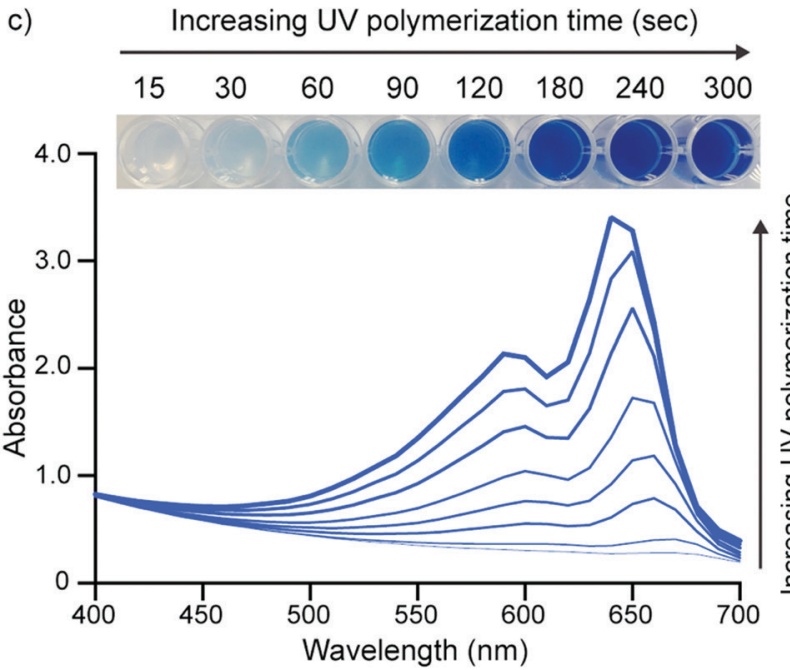

e)
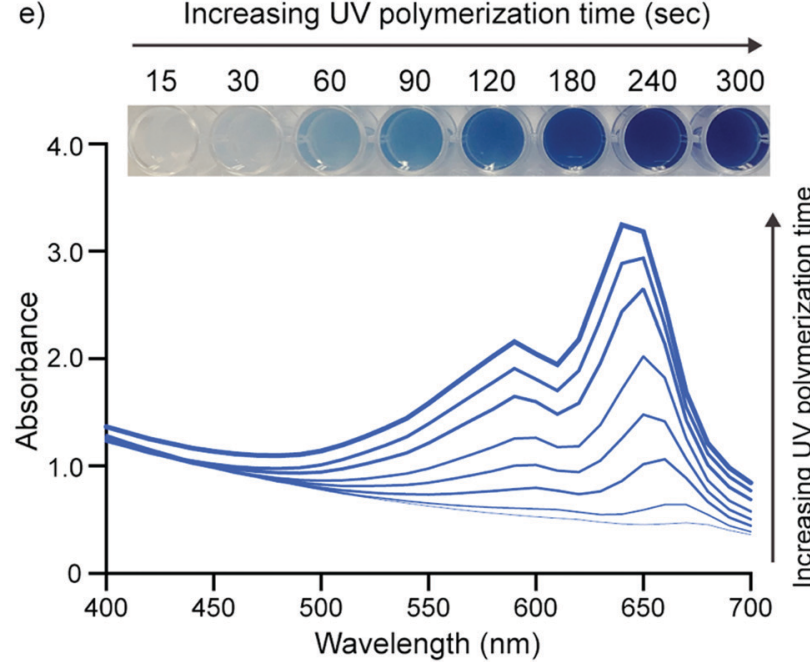

b)

Increasing UV polymerization time (sec)

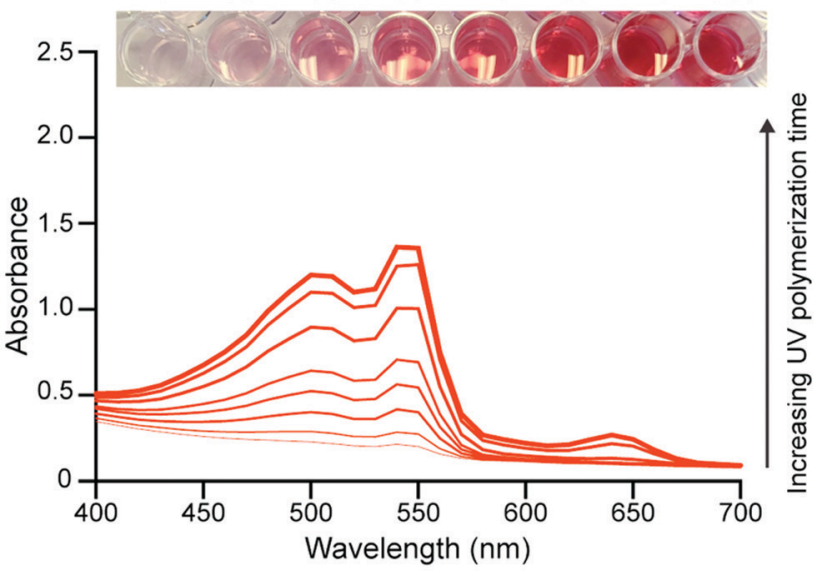

d)
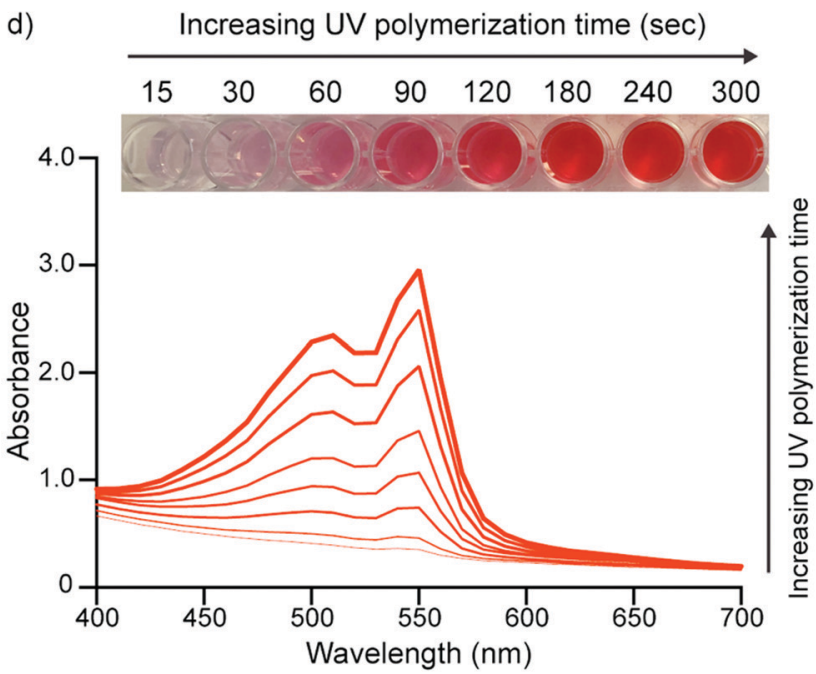

f)
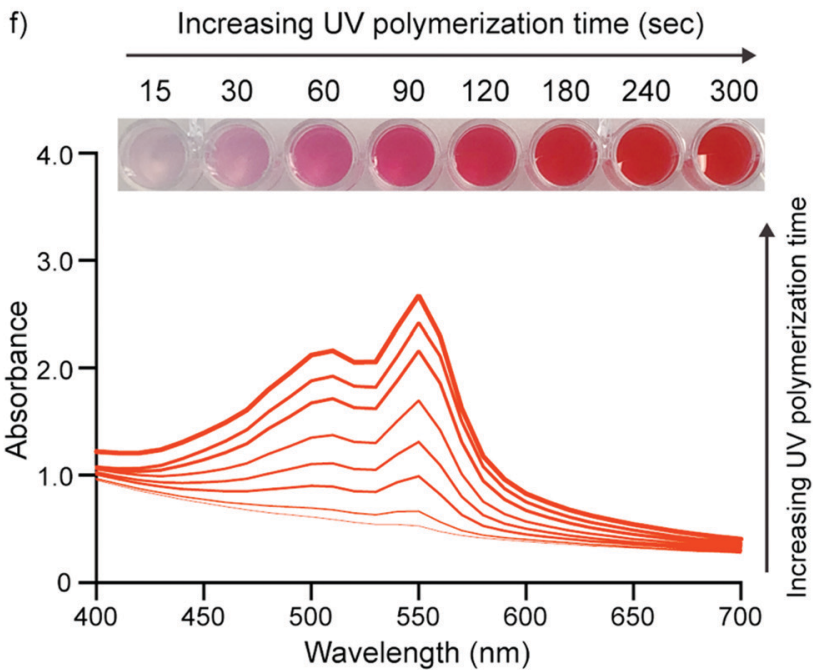

Fig. 5 UV-vis absorption spectra of PDA prepared with different UV irradiation time. Red-phase PDA were obtained by incubating blue-phase PDA at $65^{\circ} \mathrm{C}$. (a), (b) PCDA, (c), (d) TCDA, and (e), (f) HCDA. 
between $100 \mathrm{~nm}$ and $300 \mathrm{~nm}$ are large unilamellar vesicles (LUVs), and particles with size $\geq 500 \mathrm{~nm}$ are giant unilamellar vesicles (GUVs). ${ }^{19}$ Based on these classifications, PCDA, TCDA, and HCDA formed LUVs.

The particle charge ranged from $-26.9 \mathrm{mV}$ to $-32.2 \mathrm{mV}$ (Table 1). The negative surface charge of the PDA vesicles is from partial dissociation of neutral carboxylic head groups into negatively charged carboxylate head groups. Short chain carboxy terminated acids, such as the monomers used in this study, are known to have solution $\mathrm{p} K_{\mathrm{a}}$ of $\sim 4.8 .^{20}$ However, colloids and membrane structures constructed from carboxy terminated constituents are known to exhibit different ionization equilibria with $\mathrm{p} K_{\mathrm{a}} \mathrm{s}$ that differ by several orders of magnitude depending on particle structure and packing density. ${ }^{20}$ All monomers investigated in this study produced particles with a zeta potential of sufficient magnitude to keep the particles suspended in solution.

Once PDA particles were formed, they were photopolymerized by $254 \mathrm{~nm}$ UV light to yield blue-phase PDA. UV-vis spectroscopy indicated that increasing levels of exposure to UV light increased the color intensity of the blue-phase PDA for all monomers. This is illustrated by the increasing absorbance at the characteristic wavelength of $640 \mathrm{~nm}$ (Fig. 5a, c and e). This behavior is expected and is attributed to an increase in the degree of polymerization between neighboring DA monomers upon increased UV exposure. ${ }^{21-23}$ Okada et al. observed similar results when photopolymerizing PDA vesicles made from monomers of different sizes and head groups. ${ }^{17}$ Upon exposure to high temperature $\left(65^{\circ} \mathrm{C}\right)$, the PDA vesicles exhibited a characteristic blue to red color transition that could be easily detected by the naked eye and a shift in absorbance peak from $640 \mathrm{~nm}$ to $540 \mathrm{~nm}$ (Fig. 5b, d, and f).

To verify and demonstrate the functionality of the PDA formed using the solvent injection method, we employed them as a colorimetric sensor to detect ammonia gas. Ammonia gas is corrosive and a harmful irritant which can have serious health implications. Ammonia is also an indicator of food spoilage $^{21}$ and a disease biomarker. ${ }^{24}$ The detection of ammonia via carboxy-terminated PDA vesicles has been documented in various contexts and formats. ${ }^{21,25,26}$ Ammonia is a strong base and increases the $\mathrm{pH}$ of the PDA environment which encourages dissociation of carboxylic acid head groups. The negatively charged carboxylate head groups repel each other and induce structural rearrangement in the vesicle that produces a blue to red color change. ${ }^{20}$ When we exposed PDA composed of PCDA, TCDA, or HCDA to ammonia gas at $2000 \mathrm{ppm}$ in a sealed chamber, the solutions underwent a complete blue-to-red color change at $t=90 \mathrm{~min}, 80 \mathrm{~min}$, and $60 \mathrm{~min}$, respectively. The shorter the alkyl chain length of the monomer, the shorter exposure time required to induce a complete color change. This means that the system can be engineered for greater sensitivity by the selection of shorter chain length monomers. This phenomenon has been documented in systematic studies of the impact of monomer characteristics on sensing properties ${ }^{18,27-30}$ and has been demonstrated in volatile organic compound detection using PDA. ${ }^{31}$ We then subjected PDA composed of

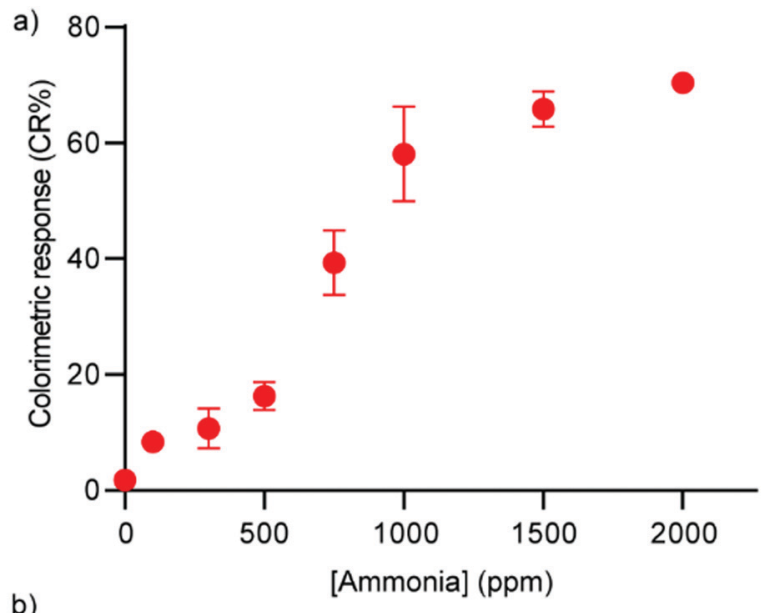

b)

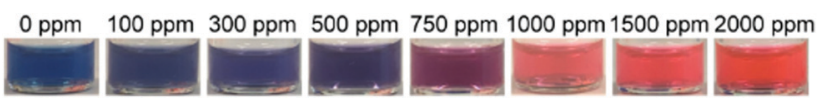

Fig. 6 (a) Colorimetric response and (b) photographs of HCDA vesicle solutions when exposed to varying concentration of ammonia. $n=3$, values represent mean \pm standard deviation.

HCDA to different ammonia concentrations (0-2000 ppm) (Fig. 6a). The resulting color change was detectable by the naked eye when the PDA solution was exposed to ammonia gas more than 500 ppm (Fig. 6b). These results indicate that the PDA particles formed using the solvent injection method maintain their characteristic functional activity upon exposure to ammonia.

To demonstrate the capability of the solvent injection method to synthesize PDA vesicles for large scale production, experiments on fabricating $10 \mathrm{ml}, 100 \mathrm{ml}$, and $250 \mathrm{ml} \mathrm{HCDA}$ vesicle solutions were conducted. A study on the particle size and morphology by DLS and TEM was also conducted to investigate the effect of different scale production on vesicle formation. According to Table 2, HCDA vesicles synthesized under different scales had a similar (within 6\% error) characteristic size with an average diameter of $\sim 280 \mathrm{~nm}$ and a narrow size distribution (PDI $\sim 0.1$ ). The particles had a relatively similar surface charge $(\sim-26 \mathrm{mV})$. TEM analysis also confirmed the morphology of HCDA vesicles being rectangular in shape when synthesized on a $250 \mathrm{ml}$ batch volume (Fig. 7). Therefore, it is reasonable to conclude that the solvent injection method is suitable to be employed for larger scale PDA vesicle production. After four months, negligible change in size distribution and zeta potential of the PDA vesicles was observed (see ESI $\dagger$ ), demonstrating the long-term stability of the PDA vesicles formed via the solvent injection method.

Table 2 Size (from the intensity plot), polydispersity index, and surface charge of PDA composed of HCDA formed under different scales

\begin{tabular}{llll}
\hline Fabrication volume & Diameter $(\mathrm{nm})$ & PDI & \multicolumn{2}{c}{ Zeta potential $(\mathrm{mV})$} \\
\hline $10 \mathrm{ml}$ & $281.9 \pm 91.2$ & $0.072 \pm 0.027$ & $-26.7 \pm 6.7$ \\
$100 \mathrm{ml}$ & $303.8 \pm 109.9$ & $0.144 \pm 0.015$ & $-24.6 \pm 7.1$ \\
$250 \mathrm{ml}$ & $264.6 \pm 89.0$ & $0.146 \pm 0.088$ & $-29.1 \pm 7.1$
\end{tabular}




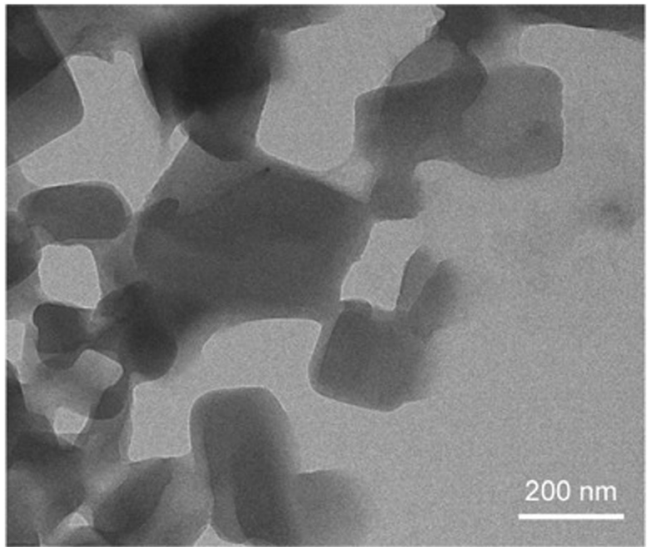

Fig. 7 A TEM micrograph of PDA composed of HCDA synthesized using the solvent injection method on a $250 \mathrm{ml}$ scale.

\section{Conclusions}

In conclusion, we have presented the first application of the solvent injection method to successfully synthesize PDA particles from a range of DA monomers with different alkyl chain length and alky spacer length. The formation of self-assembled particles similar to those formed by the film hydration method was confirmed by a study on particle size and morphology using DLS, zeta potential measurements, and TEM imaging. It was found that long chain length monomers formed smaller particles and there exists a relationship between monomer properties and particle morphology. The functionality of the PDA was demonstrated through reactivity with ammonia. PDA vesicles composed of HCDA showed color changes detectable by the naked eye when subjected to ammonia gas $>500 \mathrm{ppm}$. The solvent injection method can be applied to larger scale vesicle production $(0.25 \mathrm{~L})$. Compared with the conventional thin film hydration method, the solvent injection technique does not require secondary procedures such as extrusion, sonication, or high-pressure homogenization. PDAs have practical applications in diverse fields, including sensors, drug delivery, and tissue engineering. The ability to perform large-scale manufacturing of PDA structures could accelerate the translational development of these materials.

\section{Conflicts of interest}

There are no conflicts of interest to declare.

\section{Acknowledgements}

M. W. acknowledges support from the Australian Government Research Training Program Scholarship. K. L. acknowledges support from the National Health and Medical Research Council Career Development Fellowship (NHMRC APP1163786) and the UNSW Scientia Fellowship. R. C. acknowledges support from the National Health and Medical Research Council Emerging Leadership Investigator Grant (NHMRC EL2 APP1173428), the Australian Research Council Discovery Early Career Researcher Award (ARC DECRA DE170100068), and the UNSW Scientia Fellowship.
This research used the facilities at the Mark Wainwright Analytical Centre Electron Microscope Unit at UNSW.

\section{References}

1 M. Weston, A. D. Tjandra and R. Chandrawati, Polym. Chem., 2020, 11, 166-183.

2 J. T. Wen, J. M. Roper and H. Tsutsui, Ind. Eng. Chem. Res., 2018, 57, 9037-9053.

3 S. Lee, J. Y. Kim, X. Chen and J. Yoon, Chem. Commun., 2016, 52, 9178-9196.

4 M. Weston, F. Mazur and R. Chandrawati, Smart Sensors Environ. Med. Appl., 2020, 81-102.

5 J. Huo, Q. Deng, T. Fan, G. He, X. Hu, X. Hong, H. Chen, S. Luo, Z. Wang and D. Chen, Polym. Chem., 2017, 8, 7438-7445.

6 E. Lebègue, C. Farre, C. Jose, J. Saulnier, F. Lagarde, Y. Chevalier, C. Chaix and N. Jaffrezic-Renault, Sensors, 2018, 18, 1-16.

7 L. H. Nguyen, F. Oveissi, R. Chandrawati, F. Dehghani and S. Naficy, ACS Sens., 2020, 5, 1921-1928.

8 M. Weston, R. P. Kuchel, M. Ciftci, C. Boyer and R. Chandrawati, J. Colloid Interface Sci., 2020, 572, 31-38.

9 M. Weston, R. P. Kuchel and R. Chandrawati, Macromol. Rapid Commun., 2020, 2000172.

10 A. D. Bangham, M. M. Standish and J. C. Watkins, J. Mol. Biol., 1965, 13, 238-252.

11 S. Batzri and E. D. Korn, Biochim. Biophys. Acta, Biomembr., 1973, 298, 1015-1019.

12 A. S. Domazou and P. Luigi Luisi, J. Liposome Res., 2002, 12, 205-220.

13 A. Wagner, K. Vorauer-Uhl, G. Kreismayr and H. Katinger, J. Liposome Res., 2002, 12, 259-270.

14 P. Gentine, A. Bubel, C. Crucifix, L. Bourel-Bonnet and B. Frisch, J. Liposome Res., 2012, 22, 18-30.

15 J. M. H. Kremer, M. W. Van der Esker, C. Pathmamanoharan and P. H. Wiersema, Biochemistry, 1977, 16, 3932-3935.

16 M. Pons, M. Foradada and J. Estelrich, Int. J. Pharm., 1993, 95, 51-56.

17 S. Okada, S. Peng, W. Spevak and D. Charych, Acc. Chem. Res., 1998, 31, 229-239.

18 C. Khanantong, N. Charoenthai, T. Phuangkaew, F. Kielar, N. Traiphol and R. Traiphol, Colloids Surf., A, 2018, 553, 337-348.

19 S. E. Ahmed, H. G. Moussa, A. M. Martins, M. H. Al-Sayah and G. A. Husseini, Curr. Cancer Drug Targets, 2015, 15, 282-313.

20 S. J. Kew and E. A. H. Hall, Anal. Chem., 2006, 78, 2231-2238. 21 L. H. Nguyen, S. Naficy, R. McConchie, F. Dehghani and R. Chandrawati, J. Mater. Chem. C, 2019, 7, 1919-1926.

22 D. H. Kang, H.-S. Jung, J. Lee, S. Seo, J. Kim, K. Kim and K.-Y. Suh, Langmuir, 2012, 28, 7551-7556.

23 N. Traiphol, K. Faisadcha, R. Potai and R. Traiphol, J. Colloid Interface Sci., 2015, 439, 105-111.

24 S. A. Kharitonov and P. J. Barnes, Biomarkers, 2002, 7, 1-32. 25 G. S. Lee, C. Lee, H. Choi, D. J. Ahn, J. Kim, B. P. Gila, C. R. Abernathy, S. J. Pearton and F. Ren, Phys. Status Solidi, 2007, 204, 3556-3561. 
26 S. Park, G. S. Lee, C. Cui and D. J. Ahn, Macromol. Res., 2016, 24, 380-384.

27 N. Phonchai, C. Khanantong, F. Kielar, R. Traiphol and N. Traiphol, ACS Appl. Nano Mater., 2019, 2, 4489-4498.

28 A. Kamphan, C. Khanantong, N. Traiphol and R. Traiphol, J. Ind. Eng. Chem., 2017, 46, 130-138.
29 A. Chanakul, R. Traiphol and N. Traiphol, Colloids Surf., A, 2016, 489, 9-18.

30 A. Chanakul, N. Traiphol, K. Faisadcha and R. Traiphol, J. Colloid Interface Sci., 2014, 418, 43-51.

31 H. Jiang, Y. Wang, Q. Ye, G. Zou, W. Su and Q. Zhang, Sens. Actuators, B, 2010, 143, 789-794. 